\title{
Factores de riesgo relacionados con la conversión de colecistectomía laparoscópica a colecistectomía abierta en una Unidad de Cirugía Mayor Ambulatoria en un periodo de cinco años
}

\author{
Identify the risk factors related to the conversion of \\ laparoscopic cholecystectomy to open cholecystectomy in a \\ Major Ambulatory Surgery Unit in a period of five years \\ Guillermo López Espinosa, ${ }^{*}$ Omar Alberto Paipilla Monroy, \\ Samantha Lucia López Gómez, ${ }^{\ddagger}$ Raúl Sergio González Ramírez ${ }^{\S}$
}

Palabras clave: Colecistectomía laparoscópica, conversión, factores de riesgo.

Keywords: Laparoscopic cholecystectomy, conversion, risk factors.

\footnotetext{
${ }^{*}$ Cirujano General,
} Hospital General ISESALUD y UNEME Tijuana. ₹ Médico Pasante en servicio social. $\S$ Investigador en el Colegio de la Frontera Norte.

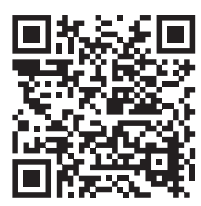

\section{RESUMEN}

La colecistectomía laparoscópica se ha convertido en el estándar de oro para el manejo de la colecistitis litiásica, por lo que conocer los factores de riesgo para la conversión a cirugía abierta podría prepararnos para la estrategia de intervención quirúrgica en una unidad de cirugía mayor ambulatoria. Objetivo: Determinar los principales factores de riesgo para conversión de colecistectomía laparoscópica a colecistectomía abierta. Material y métodos: Estudio retrospectivo observacional en 675 pacientes intervenidos, se evaluaron causas de conversión y características de los pacientes que requirieron la conversión; se describió con estadísticas descriptivas y análisis estadístico tipo prueba de $\chi^{2}$ y Fisher, además, se realizó un modelo de regresión logística para la probabilidad de conversión a cirugía abierta. Resultados: Los factores de riesgo para conversión correspondieron a hombres en $20.8 \%$, p < 0.05; pared vesicular engrosada $18.3 \%, \mathrm{p}<0.01$; bilirrubinas $52.2 \%$, $\mathrm{p}$ $<0.001$; colédoco con dilatación 37.0\%, $\mathrm{p}<0.01$; primer modelo de regresión logística con hombres 1.9 con $\mathrm{p}<0.05$ y en el segundo modelo grosor de pared vesicular $3.228 \mathrm{y}$ tamaño de colédoco 3.199 con $\mathrm{p}<0.001$. Conclusiones: Significativo para conversión en el género masculino, pared vesicular engrosada, dilatación de colédoco y bilirrubinas elevadas. La edad, duración del cuadro clínico, antecedentes de cirugía abdominal, obesidad y leucocitosis no mostraron relevancia significativa estadística. La tasa de conversión fue de $2.6 \%$ en el periodo de cinco años, similar a la reportada en otros estudios.

\section{ABSTRACT}

Laparoscopic cholecystectomy has become the gold standard for the management of lithiasic cholecystitis, so knowing the risk factors for conversion to open surgery could prepare us for the strategy of surgical intervention in an ambulatory major surgery unit. Objective: To determine the main risk factors for the conversion of laparoscopic cholecystectomy to open cholecystectomy. Material and methods: Retrospective, observational study in 675 patients undergoing surgery, evaluating causes of conversion, characteristics of patients that required conversion; describing with descriptive statistics, and statistical analysis such as $\chi^{2}$ test and Fisher, logistic regression model for probability of conversion to open surgery. Results: Risk factors for conversion, males with $20.8 \%, p<0.05$; thickened vesicular wall $18.3 \%, p<0.01$; bilirubin 52.2\%, $p<0.001$; common bile duct 37.0\%, $p$ $<0.01$; first logistic regression model, men 1.9 with $p<$ 0.05 in the second model vesicular wall thickness 3.228 and common bile duct size 3.199 with $p<0.001$. Conclusions: The male sex, thickened vesicular wall, dilation of common bile duct and elevated bilirubin were significant. Age, duration of the clinical picture and history of abdominal surgery, obesity and leukocytosis showed no significant statistical significance. The $2.6 \%$ conversion rate in the 5-year period is within the global statistical parameters.

Citar como: López EG, Paipilla MOA, López GSL, González RRS. Factores de riesgo relacionados con la conversión de colecistectomía laparoscópica a colecistectomía abierta en una Unidad de Cirugía Mayor Ambulatoria en un periodo de cinco años. Cir Gen. 2020; 42 (3): 191-196. https://dx.doi.org/10.35366/99958 


\section{INTRODUCCIÓN}

$\mathrm{L}$ a colecistectomía es uno de los procedi$ـ$ mientos quirúrgicos más realizados en el mundo, el abordaje por laparoscopia aumentó en este procedimiento, siendo el estándar de oro para el manejo de colecistitis litiásica. ${ }^{1}$ La colecistectomía laparoscópica tiene ventajas para el paciente, por lo que identificar factores preoperatorios puede predecir la dificultad del procedimiento, y permitirá discutir la probabilidad de conversión o evaluar la estrategia de manejo en los pacientes con alto riesgo durante su manejo de manera ambulatoria. ${ }^{2}$

Los factores de riesgo observados por la Sociedad Americana de Cirujanos Gastrointestinales y Endoscópicos (Society of American Gastrointestinal and Endoscopic Surgeons [SAGES]) incluyen: colecistitis aguda, engrosamiento de la pared vesicular, edad avanzada, género masculino, obesidad, hemorragia, lesión de conducto biliar y coledocolitiasis; ; ${ }^{3,4}$ en otros estudios incluyen cirugías abdominales previas, cuenta total de leucocitos elevada, impactación de lito en la bolsa de Hartmann, colección pericolecística, tamaño y número de litos, anormalidades de la anatomía, fibrosis hepática y anormalidades de pruebas de función hepática. ${ }^{5,6}$ La decisión de conversión a un procedimiento abierto se basa en un análisis intraoperatorio, observando la claridad de la anatomía, las habilidades y confort para continuar con el procedimiento, nivel II grado A, SAGES Guidelines 2010.2,4

De dos a $15 \%$ de los pacientes intervenidos de colecistectomía laparoscópica requieren una conversión a cirugía abierta, de modo que cambia el curso de la evolución del paciente; dentro de los factores de riesgo de conversión el género masculino se considera un factor elevado. Una explicación por la cual se presenta como factor de riesgo a los hombres es que tardan más en buscar ayuda y por ello se presentan con mayor alteración del cuadro al ser intervenidos quirúrgicamente. 2,5,7,8

En el Centro Médico ABC de la Ciudad de México en 2005 se estudiaron 1,843 pacientes sometidos a colecistectomía laparoscópica, tuvieron una tasa de conversión de 2.7\%, 12 casos por sangrado transoperatorio $(>600$ $\mathrm{cm}^{3}$ ), en los 39 casos restantes la principal causa de conversión fue la presencia de adherencias e imposibilidad para identificar las estructuras anatómicas, la segunda causa fue dificultad técnica (incapacidad de explorar la vía biliar laparoscópicamente), la única variable significativa fue la edad avanzada. ${ }^{9}$ En 2016 en un artículo de revisión se hizo un análisis sobre los factores que influyen en la intervención de la colecistectomía laparoscópica ambulatoria, se analizaron 25 años desde su implementación, dentro de los factores predictivos preoperatorios que ellos encuentran son: un paciente mayor de 65 años predice mayor probabilidad de falla; incremento en el tiempo quirúrgico por encontrar complicaciones intraoperatorias; colecistitis aguda que presenta pared engrosada en el ultrasonido, lo que aumenta el triple de hospitalizaciones postquirúrgicas; antecedente de historia biliar complicada por coledocolitiasis, la cual necesita otro tipo de intervención como una colangiopancreatografía retrógrada endoscópica. ${ }^{10}$

Roman Kidwai menciona en 2016 que pacientes mayores de 50 años presentaron un rango de $50 \%$ de conversión comparados con los menores de 50 años. ${ }^{11}$

La conversión de cirugía laparoscópica a cirugía abierta no es un fracaso, sino un acierto del cirujano al enfrentar dificultades técnicas en la visualización de las estructuras anatómicas o la necesidad de controlar una hemorragia. ${ }^{12}$

Por tal motivo, el objetivo general es determinar los principales factores de riesgo en nuestra unidad para conversión de colecistectomía laparoscópica a colecistectomía abierta que se realizó en un periodo de cinco años. Además, conocer los factores de riesgo preoperatorios de conversión en aquellos que requieren colecistectomía puede ayudarnos a identificar mejor a estos pacientes de alto riesgo y proponer las estrategias de manejo que se le ofrecerán.

\section{MATERIAL Y MÉTODOS}

Es un estudio retrospectivo, observacional y descriptivo realizado en la unidad de cirugía ambulatoria mayor (UNEME) en un periodo de cinco años, de enero de 2013 a diciembre de 2017. Se incluyeron a todos los pacientes programados en forma electiva para colecistectomía laparoscópica ambulatoria con ex- 


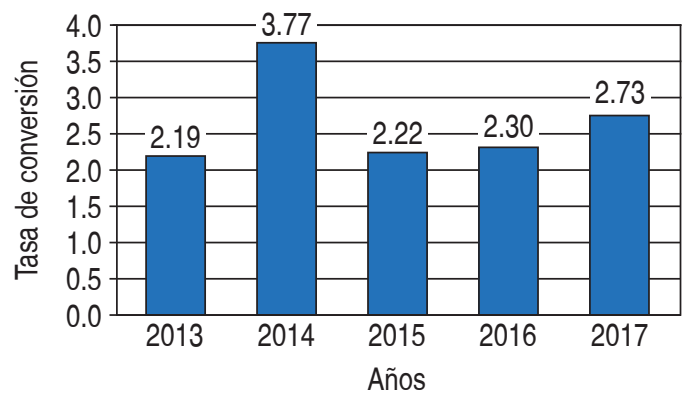

Figura 1: Tasa de conversión por año.

Elaboración propia de acuerdo con datos consultados.

pediente completo, laboratorios, ultrasonido y nota postquirúrgica completos. Después de la exclusión de expedientes el estudio se realizó en 675 pacientes de un total de 3,317 intervenidos de colecistectomía laparoscópica.

Se evaluaron variables de edad, género, índice de masa corporal (IMC), patologías concomitantes, antecedentes de cirugía abdominal, tiempo de evolución, parámetros ultrasonográficos, resultados de laboratorio preoperatorios, causas de conversión, diagnóstico de conversión y año de la cirugía.

Para su análisis se realizó estadística descriptiva para frecuencias y promedios, pruebas de $\chi^{2}$ y exacta de Fisher para tabulaciones cruzadas de los factores de riesgo asociados con la conversión a cirugía abierta. Se estimaron modelos de regresión logística para la probabilidad de conversión a cirugía abierta. Se utilizó el Programa SPSS Statistics 24 para realizar el análisis estadístico.

Tabla 1: Distribución de los pacientes conforme a la conversión de cirugía por grosor de pared vesicular. UNEME, Tijuana 2013-2017.

\begin{tabular}{lcrrr} 
& & \multicolumn{2}{c}{$\begin{array}{c}\text { Grosor de pared } \\
\text { vesicular* (\%) }\end{array}$} & \\
\cline { 3 - 4 } & & Normal & Engrosada & Total (\%) \\
\cline { 3 - 4 } & No & 92.00 & 81.70 & 88.50 \\
Convertidas & Sí & 8.00 & 18.30 & 11.50 \\
Total & & 100.00 & 100.00 & 100.00 \\
\hline
\end{tabular}

$* p<0.001$, siendo significativo el grosor de la pared vesicular para la conversión. Elaboración propia de acuerdo con datos consultados.

\section{RESULTADOS}

De los 675 pacientes $85.8 \%$ fueron mujeres y $14.2 \%$ hombres; $83.4 \%$ fueron menores de 55 años y $16.6 \%$ mayores de 55 años; $9.8 \%$ tuvieron diabetes, 53.5\% antecedentes de cirugía abdominal, $43.4 \%$ obesidad y $55.8 \%$ un cuadro clínico de seis meses. Dentro de los parámetros de ultrasonido el grosor de pared vesicular en $66.3 \%$ fue normal y $33.7 \%$ tuvieron la pared engrosada (se consideró $>3$ $\mathrm{mm}$ como engrosamiento de pared vesicular); 94\% tuvo dilatación de colédoco (se emplearon parámetros $>6 \mathrm{~mm}$ como colédoco dilatado por ultrasonido); $98.1 \%$ tuvo presencia de litos en ultrasonido. Dentro de parámetros de laboratorio $96 \%$ tuvo normales las bilirrubinas y $4.0 \%$, elevadas; los leucocitos en $89.2 \%$ fueron normales y en $10.8 \%$ elevados.

La tasa de conversión por año se ha mantenido constante, hubo una elevación en el año 2014 con $3.7 \%$ y se obtuvo una tasa total por el periodo de enero de 2013 a diciembre de 2017 de 2.6\%, la cual está dentro de los parámetros mundiales del rango de conversión a cirugía abierta. ${ }^{1,2}$

Se analizaron por medio de tablas cruzadas las variables estudiadas con respecto a los pacientes convertidos, los resultados se observan en la Figura 1.

La conversión de cirugía la requirieron $20.8 \%$ de los hombres y $11.7 \%$ de las mujeres con $p<0.05 ; 8.0 \%$ tuvo la pared vesicular normal en ultrasonido y $18.3 \%$ tuvo la pared engrosada con $\mathrm{p}<0.001$; con respecto a las bilirrubinas $10.7 \%$ las tuvieron normales y $52.2 \%$, elevadas con $p<0.001 ; 9.3 \%$ no tuvo dilatación de colédoco mientras que $37 \%$ sí con $p<0.01 ; 11.6 \%$ tuvieron antecedentes de cirugía abdominal; $17 \%$ fueron mayores de 55 años; $13.6 \%$ tuvieron antecedentes de diabetes; $16.7 \%$, leucocitos elevados; $14 \%$ con cuadro clínico de entre uno y dos años; $14.9 \%$ tuvieron obesidad (Tablas 1 a 3).

Se realizaron dos modelos de regresión logística para la probabilidad de conversión a cirugía abierta y se obtuvo lo siguiente: $p<$ 0.05, $\mathrm{p}<0.001$.

En el primer modelo con variables demográficas se obtuvo que el género masculino 
Tabla 2: Distribución de los pacientes conforme a la conversión de cirugía por diabetes. UNEME, Tijuana 2013-2017.

\begin{tabular}{lcrrr} 
& & \multicolumn{2}{c}{ Diabetes (\%) } & \\
\cline { 3 - 4 } & & \multicolumn{1}{c}{ No } & \multicolumn{1}{c}{ Sí } & Total (\%) \\
\hline \multirow{2}{*}{ Convertidas } & No & 87.00 & 86.40 & 87.00 \\
\multirow{2}{*}{ Total } & Sí & 13.00 & 13.60 & 13.00 \\
& & 100.00 & 100.00 & 100.00 \\
\hline
\end{tabular}

Elaboración propia de acuerdo con datos consultados.

tuvo una probabilidad de 1.9 con $p<0.05$, y en el segundo modelo se agregaron variables ultrasonográficas de grosor de pared con una probabilidad de 3.228, colédoco 3.199 con $p$ $<0.001$ (Tabla 4).

\section{DISCUSIÓN}

Los factores de riesgo para la conversión a colecistectomía abierta han mostrado ser los mismos para una colecistectomía difícil, es muy importante tener los datos completos del paciente, su historia clínica, laboratorios y estudios de gabinete como el ultrasonido.

Una de las limitaciones de nuestro estudio fue que los ultrasonidos no tenían una forma de medición estandarizada, ya sea por medio público o privado todos tienen información con parámetros inconstantes (todos los ultrasonidos no son de la unidad), por lo que es importante

Tabla 3: Distribución de los pacientes conforme a la conversión de cirugía por tamaño de colédoco. UNEME, Tijuana 2013-2017.

\begin{tabular}{lcccr} 
& \multicolumn{2}{c}{ Colédoco* (\%) } & \\
\cline { 3 - 4 } & & No dilatación & Sí dilatación & Total (\%) \\
\hline \multirow{2}{*}{ Convertidas } & No & 90.70 & 63.00 & 89.10 \\
& Sí & 9.30 & 37.00 & 10.90 \\
Total & & 100.00 & 100.00 & 100.00 \\
\hline
\end{tabular}

$* \mathrm{p}<0.001$, siendo significativo la dilatación de colédoco para la conversión. Elaboración propia de acuerdo con datos consultados. conocer bien los datos de la pared vesicular. El ultrasonido preoperatorio se ha usado como un método para indicar las dificultades técnicas y predecir la conversión potencial. ${ }^{13,14}$

En nuestro estudio se utilizó el grosor de la pared vesicular significativo arriba de $3 \mathrm{~mm}$, ya que es un signo radiológico de colecistitis aguda con cambios inflamatorios, ${ }^{4,15}$ el rango del conducto biliar común es a partir de 4-8 $\mathrm{mm}, 10,13$ al igual que el tamaño del colédoco dilatado, arriba de $6 \mathrm{~mm}$ se ha reportado como colecistectomía difícil; ${ }^{16}$ además, no se ha visto significancia en el número de piedras y riesgo de conversión. ${ }^{1,2}$ Con lo mencionado anteriormente, se observa que estos son datos de un cuadro agudo: el primero con inflamación e induración del tejido, lo que hace difícil la disección del triángulo de Calot por proceso inflamatorio ${ }^{7,8} \mathrm{y}$ el segundo es parte de los factores predictores de coledocolitiasis, lo cual adhiere otro problema a nuestro cuadro inicial. ${ }^{17}$

Dentro de los resultados de los pacientes intervenidos podemos advertir que la unidad se encuentra dentro de los parámetros mundiales de conversión. Los parámetros de conversión utilizados para nuestro análisis fueron: el género masculino, ya que se ha visto mayor incidencia de conversión a cirugía abierta que en mujeres; grosor de pared vesicular $>3 \mathrm{~mm}$; dilatación de colédoco $>6 \mathrm{~mm}$ y presencia o no de litos por ultrasonido; elevación de bilirrubinas $>1.5 \mathrm{mg} /$ $\mathrm{dl}$; leucocitosis $>11,000 \mathrm{~mm} / \mathrm{dl}$; antecedentes de cirugía abdominal; edad avanzada mayor a 55 años; diabetes; índice de masa corporal y cuadro clínico mayor de seis meses de evolución. ${ }^{18-20}$

Con respecto a los motivos de conversión los más prevalentes fueron los siguientes: adherencias, dilatación de vía biliar y dificultad para realizar la visión crítica de Strasberg; estos resultados que se obtuvieron tienen relación con otros estudios en los que también tuvieron como motivos una dificultad para la disección del triángulo de Calot y hemorragia transoperatoria, lo que oscurece de primera instancia el campo operatorio sin poder identificar bien nuestros puntos de seguridad anatómicos. ${ }^{1,5,21}$ La explicación a la incidencia de estos factores pudiera ser que el curso prolongado de los síntomas desarrolle un mayor proceso inflamatorio severo y progresivo, lo que impide el término exitoso de la cirugía laparoscópica. ${ }^{22}$ 


\begin{tabular}{|c|c|c|c|}
\hline \multirow[b]{2}{*}{ Variable } & & \multirow{2}{*}{$\begin{array}{c}\text { Demográfico } \\
\text { Modelo } 1\end{array}$} & \multirow{2}{*}{$\begin{array}{r}\text { Ultrasonido } \\
\text { Modelo } 2\end{array}$} \\
\hline & & & \\
\hline Género & $\begin{array}{c}\text { Hombre } \\
\text { (Ref. mujer) }\end{array}$ & $1.9 *$ & 1.13 \\
\hline Edad & $\begin{array}{c}>55 \\
(\text { Ref. }<55)\end{array}$ & 1.355 & \\
\hline Grosor de pared & $\begin{array}{c}>3 \mathrm{~mm} \\
(\text { Ref. }<3 \mathrm{~mm})\end{array}$ & & $3.228^{\ddagger}$ \\
\hline Colédoco & $\begin{array}{c}>6 \mathrm{~mm} \\
\text { (Ref. }<6 \mathrm{~mm})\end{array}$ & & $3.199^{\ddagger}$ \\
\hline Constante & & 0.241 & $0.021^{\ddagger}$ \\
\hline \multicolumn{4}{|c|}{$\begin{array}{l}\text { Modelo 1: variables demográficas, el género tuvo una } \mathrm{p}<0.05^{*} \text { significativa en } \\
\text { relación a la conversión. } \\
\text { Modelo 2: variables ultrasonográficas, tuvieron una } \mathrm{p}<0.001^{\ddagger} \text { significativa en } \\
\text { relación a la conversión. } \\
\text { Elaboración propia de acuerdo con datos consultados. }\end{array}$} \\
\hline
\end{tabular}

En estos factores observamos que elevan el riesgo de conversión, ya que lleva a otras dificultades y destrezas durante el acto operatorio, por lo que seguir evaluando la eficacia de estos instrumentos de diagnóstico para identificar factores de riesgo, así como la dificultad aumentada por estos factores de las cirugías intervenidas nos ayuda a continuar estudiando las opciones de manejo que se tienen y en casos necesarios planear la estrategia de manejo del paciente.

\section{CONCLUSIONES}

Los resultados de este estudio serán de apoyo para valorar a los pacientes antes de ser intervenidos de colecistectomía por vía laparoscópica y así tener en cuenta qué paciente tiene riesgo de ser convertido a procedimiento abierto y con esto llevar a mayor tiempo de hospitalización y más uso de recursos para el paciente y las unidades hospitalarias.

\section{REFERENCIAS}

1. Overby W, Apelgren KN. SAGES guidelines for the clinical application of laparoscopic biliary tract surgery. Surg Endosc. 2010; 24: 2368-286.
2. Kassa $V$, Jaiswal R. Assessment of risk factors for difficult surgery in laparoscopic cholecystectomy. IMJ. 2017; 4: 258-262.

3. Shiun Yew Hu A, Menon R, Gunnarsson R, De Costa A. Risk factors for conversion of laparoscopic cholecystectomy to open surgery a systematic literature review of 30 studies. Am J Surg. 2017; 214: 920-930.

4. Tosun A, Oymen HK, Serifoglu I, Capan Y, Ozkaya E. Role of preoperative sonography in predicting conversion from laparoscopic cholecystectomy to open surgery. Eur J Radiol. 2015; 84: 346-349.

5. Ostos LJ, Perez E. Tratado de cirugía general. AMCG. 2017; 1: 1343-1350.

6. Galloso GL, Frías RA. Factores que influyen en la conversión de la colecistectomía video laparoscópica a cirugía tradicional. Rev Cubana Med Mil. 2012; 41: 352-360.

7. Hunter JG. Acute cholecystitis revisited. Ann Surg. 1998; 227: 468-469.

8. Cicero LA, Valdés FJ, Decanini MA, Golffier RC, Cicero LC, Cervantes CJ, Rojas RG. Factores que predicen la conversión de la colecistectomía laparoscópica: Cinco años de experiencia en el Centro Médico ABC. Rev Mex Cir Endoscop. 2005; 6: 66-73.

9. Rothman JP, Burcharth J. Preoperative risk factors for conversion of laparoscopic cholecystectomy to open surgery- A systematic review and meta-analysis of observational studies. Dig Surg. 2016; 33: 414-423.

10. Rashid T, Naheed A. Conversion of laparoscopic cholecystectomy into open cholecystectomy: an experience in 300 cases. Med Coll Abbottabad. 2016; 28: 116-119.

11. Ocádiz J, Salvador J. Conversión de la colecistectomía laparoscópica, más allá de la curva de aprendizaje. Acta Med. 2011; 9: 192-195.

12. Kama NA, Kologlu M. A risk score for conversion from laparoscopic to open cholecystectomy. Am J Surg. 2001; 181: 520-523.

13. Kania D. Ultrasound measurement of the Gallbladder wall thickness in the assessment of the risk of conversion from elective laparoscopic cholecystectomy to open surgery- Olkusz county experience. Pol Przegl Chir. 2016; 88: 334-345.

14. Pinto A, Reginelli A, Cagini L, Coppolino F, Stabile IA, Bracale R, et al. Accuracy of ultrasonography in the diagnosis of acute calculous cholecystitis: review of the literature. Crit Ultrasound J. 2013; 5: S11.

15. Abdulhussein B, Hussein Y, Nawar A, Al-Naggar R. Conversion rate of laparoscopic cholecystectomy to open surgery at Al Karamah Teaching Hospital, Iraq. Surgical Science. 2015; 6: 221-226.

16. Pawan L, Agarwal PN. A difficult laparoscopic cholecystectomy that requires conversion to open procedure can be predicted by preoperative ultrasonography. JSLS. 2002; 6: 59-63.

17. Maple JT. The role of endoscopy in the evaluation of suspected choledocholithiasis. SAGES. 2010; 71: 1-9.

18. Fernandes $U$, Bertozzi M. Ambulatory laparoscopic cholecystectomy is safe and cost-effective: a Brazilian single center experience. Arq Gastroenterol. 2016; 53: 103-107.

19. Bueno J, Granero P. Twenty-five years of ambulatory laparoscopic cholecystectomy. Cir Esp. 2016; 94 : 429-441. 
20. Juarez D, Escamilla A. Género masculino factor de riesgo para la colecistectomía. Cir Gen. 2006; 28: 93-96.

21. Strasberg SM, Brunt M. Rationale and use of the critical view of safety in laparoscopic cholecystectomy. J Am Coll Surg. 2010; 211: 132-138.

22. Prieto-Díaz-Chávez E, Medina-Chavez JL. Factores de riesgo para conversión de colecistectomía laparoscópica a colecistectomía abierta. Cir Gen. 2010; 32: 34-38.

Consideraciones y responsabilidad ética: Privacidad de los datos. De acuerdo a los protocolos establecidos en el centro de trabajo de los autores, estos declaran que han seguido los protocolos sobre la privacidad de datos de pacientes preservando su anonimato.

Financiamiento: No se recibió apoyo financiero para la realización de este trabajo.

Conflicto de intereses: Los autores declaran que no existe ningún conflicto de intereses en la realización del trabajo.

Correspondencia:

Guillermo López Espinosa

E-mail: glopezcirugia@gmail.com 